\title{
UNIFORM BOUNDEDNESS OF OSCILLATORY SINGULAR INTEGRALS ON HARDY SPACES
}

\author{
Leslie C. Cheng and Yibiao Pan
}

Abstract

We prove the uniform $H^{1}$ boundedness of oscillatory singular integrals with degenerate phase functions.

\section{Introduction}

In a recent paper [1], Carbery, Christ, and Wright established a series of important estimates for oscillatory integrals and sublevel sets. As one of the applications they also obtained the uniform boundedness on $L^{p}$ spaces of oscillatory singular integrals with possibly flat phase functions. The purpose of this paper is to apply their results to the study of the uniform boundedness of oscillatory singular integrals on the Hardy space $H^{1}\left(\mathbf{R}^{n}\right)$.

Oscillatory singular integral operators are singular integral operators which carry oscillatory factors in their kernels. They have arisen in many problems in harmonic analysis and related areas and have been studied extensively $([\mathbf{1 0}],[\mathbf{1 1}])$. For previous work on the boundedness on $L^{p}$ and Hardy spaces of such operators, see Phong-Stein [7], Ricci-Stein [8], Stein [10], Pan [5], [6], and Carbery-Christ-Wright [1].

Let $n \in \mathbf{N}, \varphi \in C_{0}^{\infty}\left(\mathbf{R}^{n}\right)$, and $\Phi \in C^{\infty}\left(\mathbf{R}^{n}\right)$ be a real-valued function satisfying $\nabla \Phi(0)=0$. Let $K(\cdot)$ be a Calderón-Zygmund kernel on $\mathbf{R}^{n}$, i.e. $|K(x)|+|x||\nabla K(x)| \leq A|x|^{-n}$ and $\int_{a<|x|<b} K(x) d x=0$ for $b>a>0$.

For each $\lambda \in \mathbf{R}$ we define the (localized) oscillatory singular integral operator $T_{\lambda}$ on $\mathbf{R}^{n}$ by

$$
\left(T_{\lambda} f\right)(x)=\text { p.v. } \int_{\mathbf{R}^{n}} e^{i \lambda \Phi(x-y)} K(x-y) \varphi(x-y) f(y) d y .
$$

The following was proved in $[\mathbf{6}]$ : 
Theorem A. Let $K, \varphi, \Phi$, and $T_{\lambda}$ be given as above. If $D^{\alpha} \Phi(0) \neq 0$ for some multi-index $\alpha$ with $|\alpha| \geq 2$, then there exists a positive constant $C$ such that

$$
\left\|T_{\lambda} f\right\|_{H^{1}\left(\mathbf{R}^{n}\right)} \leq C\|f\|_{H^{1}\left(\mathbf{R}^{n}\right)}
$$

for $f \in H^{1}\left(\mathbf{R}^{n}\right)$ and $\lambda \in \mathbf{R}$.

Since it follows easily from the standard theory of singular integrals that $T_{\lambda}$ is a bounded operator on $H^{1}\left(\mathbf{R}^{n}\right)$ for each $\lambda \in \mathbf{R}$, the importance of Theorem A lies in the uniformity of bounds on the operator norms $\left\|T_{\lambda}\right\|_{H^{1} \rightarrow H^{1}}$. It was also shown in [6] that (2) may not hold if $D^{\alpha} \Phi(0)$ vanishes for every $\alpha$.

Theorem B. Let $x, y \in \mathbf{R}$. Define

$$
\left(T_{\lambda} f\right)(x)=p . v . \quad \int_{|x-y| \leq 1} e^{i \lambda \Phi(x-y)} \frac{f(y)}{x-y} d y .
$$

There exists a nonconstant function $\Phi \in C^{\infty}(\mathbf{R})$ with $\Phi^{(k)}(0)=0$ for all $k=0,1,2, \ldots$, such that

$$
\sup _{\lambda \in \mathbf{R}}\left\|T_{\lambda}\right\|_{H^{1} \rightarrow H^{1}}=\infty .
$$

A phase function $\Phi$ is said to be flat at 0 if $D^{\alpha} \Phi(0)=0$ for all $\alpha$. Can the uniform boundedness of $T_{\lambda}$ 's on $H^{1}\left(\mathbf{R}^{n}\right)$ still hold when the phase function $\Phi$ is flat at 0? Inspired by the work of Carbery, Christ, and Wright we address this issue by establishing the following:

Theorem C. Let $K, \varphi, \Phi$, and $T_{\lambda}$ be given as in Theorem A. Let $\alpha$ be a multi-index with $|\alpha| \geq 3$. Suppose that at least one coordinate of $\alpha$ is strictly greater than 1 and there exist $\delta, A>0$ such that

$$
\max _{|\beta|=|\alpha|} \sup _{|x| \leq s}\left|D^{\beta} \Phi(x)\right| \leq A \inf _{s \leq|x| \leq \delta}\left|D^{\alpha} \Phi(x)\right|
$$

holds for all $s \in(0, \delta)$. Then there exists a positive constant $C$ such that

$$
\left\|T_{\lambda} f\right\|_{H^{1}\left(\mathbf{R}^{n}\right)} \leq C\|f\|_{H^{1}\left(\mathbf{R}^{n}\right)}
$$

for $f \in H^{1}\left(\mathbf{R}^{n}\right)$ and $\lambda \in \mathbf{R}$.

Condition (5) is simply Condition (8.1) of [1] in the convolutional setting. As pointed out in [1] , (5) is always satisfied if $D^{\alpha} \Phi(0) \neq 0$ and it may also be satisfied even if $\Phi$ is flat at 0 . For example, when $n=1, \Phi(x)=\eta(x) \exp \left(\omega(x) / x^{2}\right)$ satisfies (5) if $\eta$ and $\omega$ are smooth and $\eta(0) \neq 0, \omega(0)<0$.

Throughout the rest of the paper the letter $C$ will stand for a constant but not necessarily the same one in each occurrence. 


\section{Some Lemmas}

Lemma 2.1. Let $K, \varphi, \Phi$, and $T_{\lambda}$ be given as in Theorem A. Let $\alpha$ be a multi-index with $|\alpha| \geq 3$. Suppose that at least one coordinate of $\alpha$ is strictly greater than 1 and that (5) holds for some $\delta, A>0$, and every $s \in(0, \delta)$. Then for every $p \in(1, \infty)$ there exists a positive constant $C_{p}$ such that

$$
\left\|T_{\lambda} f\right\|_{L^{p}\left(\mathbf{R}^{n}\right)} \leq C_{p}\|f\|_{L^{p}\left(\mathbf{R}^{n}\right)}
$$

for $f \in L^{p}\left(\mathbf{R}^{n}\right)$ and $\lambda \in \mathbf{R}$.

Proof: Let $\psi \in C_{0}^{\infty}\left(\mathbf{R}^{n}\right)$ such that $\sum_{m \in \mathbf{Z}^{n}} \psi_{m} \equiv 1$ where $\psi_{m}(x)=$ $\psi(x-m)$. Let $T_{\lambda, m}(f)=T_{\lambda}\left(\psi_{m} f\right)$. It follows from Theorem 8.2 in [1] that the operators $\left\{T_{\lambda, m}: \lambda \in \mathbf{R}, m \in \mathbf{Z}^{n}\right\}$ are uniformly bounded on $L^{p}\left(\mathbf{R}^{n}\right)$ for $1<p<\infty$. One then obtains (7) by observing that the sets $\left\{\operatorname{supp}\left(T_{\lambda, m}(f)\right): m \in \mathbf{Z}^{n}\right\}$ have the finite overlapping property.

Let $Q^{n}=[-1 / 2,1 / 2]^{n}$. The following result is due to Carbery, Christ, and Wright $([\mathbf{1}])$.

Lemma 2.2. For $n=n^{\prime}+n^{\prime \prime}$, each $1 \leq p, q \leq \infty(p=1, q=\infty)$ and each $\beta \in(\mathbf{N} \cup\{0\})^{n}$ with at least one nonzero entry in each of $\left\{1, \ldots, n^{\prime}\right\}$ and $\left\{n^{\prime}+1, \ldots, n^{\prime \prime}\right\}$ and with at least one entry greater than 1 , there exist $\varepsilon=\varepsilon(n, \beta, p, q)>0$ and $C=C(\varepsilon, n, \beta, p, q)$ such that for every real-valued, integrable function u satisfying $D^{\beta} u \geq 1$ on $Q^{n}$, for every $\lambda \in \mathbf{R}$, the operator

$$
\left(S_{\lambda} f\right)\left(x^{\prime}\right)=\int_{Q^{n^{\prime \prime}}} e^{i \lambda u\left(x^{\prime}, x^{\prime \prime}\right)} f\left(x^{\prime \prime}\right) d x^{\prime \prime}
$$

satisfies

$$
\left\|S_{\lambda} f\right\|_{L^{q}\left(Q^{n^{\prime}}\right)} \leq C \lambda^{-\varepsilon}\|f\|_{L^{p}\left(Q^{n^{\prime \prime}}\right)} .
$$

\section{Proof of Theorem C}

Let $K, \varphi, \Phi$, and $T_{\lambda}$ be given as in Theorem C. By the atomic decomposition of $H^{1}\left(\mathbf{R}^{n}\right)$ and the characterization of $H^{1}\left(\mathbf{R}^{n}\right)$ using Riesz transforms (see [2]-[4], [11]), it suffices to show that $T_{\lambda} f$ is in $L^{1}\left(\mathbf{R}^{n}\right)$ uniformly in $\lambda$ for all atoms $f$. 
Let $\alpha$ be the multi-index such that (5) holds and set

$$
J(s)=s^{|\alpha|-1} \inf _{s \leq|x| \leq \delta}\left|D^{\alpha} \Phi(x)\right| .
$$

Let $\rho>0$ be small (say, $\rho<\delta / 2$ ), $f$ be an atom which is supported in $\rho Q^{n}$ and satisfies $\|f\|_{\infty} \leq \rho^{-n}$ and $\int_{\mathbf{R}^{n}} f(x) d x=0$. Let $\lambda>0$.

We shall first prove that

$$
\int_{|x| \geq 2 \rho}\left|T_{\lambda} f(x)\right| d x \leq C
$$

holds for some constant $C$ independent of $\lambda, \rho$, and $f$.

If $\lambda \rho \leq(J(\delta))^{-1}$, then (10) follows from

$$
\begin{aligned}
\left|T_{\lambda} f(x)\right| & \leq \frac{A}{|x|^{n}} \int_{\rho Q^{n}}\left|e^{i \lambda \Phi(x-y)}-e^{i \lambda \Phi(x)}\right||\varphi(x-y) f(y)| d y+\left|T_{0} f(x)\right| \\
& \leq \frac{A(\lambda \rho)}{|x|^{n-1}} \int_{\rho Q^{n}}|\varphi(x-y) f(y)| d y+\left|T_{0} f(x)\right|
\end{aligned}
$$

for $|x| \geq 2 \rho$. Thus we may assume that $\lambda \rho>(J(\delta))^{-1}$.

Let $t=\max \left\{J^{-1}(1 /(\lambda \rho)), 2 \rho\right\}$. For $j \geq 1$ define the operator $\mathcal{Q}_{j}$ by

$$
\left(\mathcal{Q}_{j} f\right)(x)=\chi_{[1,2]}(|x|) \int_{Q^{n}} e^{i \lambda \Phi\left(2^{j} t x-\rho y\right)} \varphi\left(2^{j} t x-\rho y\right) g(y) d y
$$

By our assumption there exist multi-indices $\alpha^{(1)}, \alpha^{(2)}$ such that $\alpha=$ $\alpha^{(1)}+\alpha^{(2)},\left|\alpha^{(2)}\right|=1$, and at least one entry of $\alpha^{(1)}$ is greater than 1 . Thus

$$
\left|D_{x}^{\alpha^{(1)}} D_{y}^{\alpha^{(2)}}\left[\Phi\left(2^{j} t x-\rho y\right)\right]\right| \geq\left[2^{j(|\alpha|-1)} \rho\right] J(t)
$$

holds for $1 \leq|x| \leq 2$ and $y \in Q^{n}$. Since the factor $\varphi\left(2^{j} t x-\rho y\right)$ is harmless, it follows from Lemma 2.2 that

$$
\left(\int_{1 \leq|x| \leq 2}\left|\mathcal{Q}_{j} g(x)\right|^{2} d x\right)^{1 / 2} \leq C\left[2^{j(|\alpha|-1)} \lambda \rho J(t)\right]^{-\varepsilon}\|g\|_{L^{2}\left(Q^{n}\right)}
$$


for some positive $\varepsilon$ independent of $\alpha, \rho, j$ and $g$. By letting $f_{\rho}(x)=$ $\rho^{n} f(\rho x)$ and rescaling, we have

$$
\begin{aligned}
\int_{|x| \geq 2 t}\left|T_{\lambda} f(x)\right| d x \leq & C \int_{|x| \geq 2 t}\left|\int_{\mathbf{R}^{n}} e^{i \lambda \Phi(x-y)} \varphi(x-y) f(y) d y\right| \frac{d x}{|x|^{n}} \\
& +\int_{|x| \geq 2 \rho} \int_{\rho Q^{n}}|K(x-y)-K(x) \| f(y)| d y d x \\
\leq & C \sum_{j \geq 1}\left\|\mathcal{Q}_{j}\left(f_{\rho}\right)\right\|_{2}+C \\
\leq & C\left[1+(\lambda \rho J(t))^{-\varepsilon} \sum_{j \geq 1} 2^{-\varepsilon(|\alpha|-1) j}\right] \leq C .
\end{aligned}
$$

Let

$$
P(x)=\sum_{|\beta| \leq|\alpha|-1}(\beta !)^{-1} D^{\beta} \Phi(0) x^{\beta}
$$

and $\Psi(x)=\Phi(x)-P(x)$. By Theorem A,

$$
\int_{\mathbf{R}^{n}}\left|\int_{\mathbf{R}^{n}} e^{i \lambda P(x-y)} K(x-y) \varphi(x-y) f(y) d y\right| d x \leq C .
$$

Thus, by (14)

$$
\begin{aligned}
& \int_{2 \rho \leq|x|<2 t}\left|T_{\lambda} f(x)\right| d x \\
& \leq \int_{2 \rho \leq|x| \leq t / 2} \int_{\rho Q^{n}}\left|e^{i \lambda(\Psi(x-y)-\Psi(x))}-1\right||K(x-y) \varphi(x-y) f(y)| d y d x+C \\
& \leq C \lambda\left(\max _{|\beta|=|\alpha|} \sup _{|x| \leq t}\left|D^{\beta} \Phi(x)\right|\right) \\
& \quad \times\left(\int_{2 \rho \leq|x| \leq t / 2}|x|^{|\alpha|-1-n} d x\right)\left(\int_{\rho Q^{n}}|y||f(y)| d y\right)+C \\
& \leq C \lambda \rho J(t)+C \leq C,
\end{aligned}
$$

where we took $t=J^{-1}(1 /(\lambda \rho))$ (otherwise $t=2 \rho$ and the integral of $\left|T_{\lambda} f(x)\right|$ on $\{2 \rho \leq|x|<2 t\}$ is trivially bounded). By combining (13) and (15) we see that (10) holds. 
By Hölder's inequality and Lemma 2.1,

$$
\int_{|x|<2 \rho}\left|T_{\lambda} f(x)\right| d x \leq C \rho^{n / 2}\|f\|_{2} \leq C .
$$

It follows from (10) and (16) that

$$
\left\|T_{\lambda} f\right\|_{1} \leq C
$$

holds for those atoms $f$ described at the beginning of our proof. By using translation and (16) (for large $\rho$ 's) we see that (17) holds for an arbitrary atom $f$. Theorem $\mathrm{C}$ is proved.

\section{References}

[1] A. Carbery, M. Christ and J. Wright, Multidimensional van der Corput and sublevel set estimates, J. Amer. Math. Soc. 12(4) (1999), 981-1015.

[2] R. R. Colfman, A real variable characterization of $H^{p}$, Studia Math. 51 (1974), 269-274.

[3] C. Fefferman and E. M. Stein, $H^{p}$ spaces of several variables, Acta Math. 129(3-4) (1972), 137-193.

[4] R. H. LatteR, A characterization of $H^{p}\left(\mathbf{R}^{n}\right)$ in terms of atoms, Studia Math. 62(1) (1978), 93-101.

[5] Y. B. PAN, Uniform estimates for oscillatory integral operators, $J$. Funct. Anal. 100(1) (1991), 207-220.

[6] Y. B. PAN, Boundedness of oscillatory singular integrals on Hardy spaces. II, Indiana Univ. Math. J. 41(1) (1992), 279-293.

[7] D. H. Phong And E. M. Stein, Hilbert integrals, singular integrals, and Radon transforms. I, Acta Math. 157(1-2) (1986), 99-157.

[8] F. RicCi And E. M. Stein, Harmonic analysis on nilpotent groups and singular integrals. I. Oscillatory integrals, J. Funct. Anal. 73(1) (1987), 179-194.

[9] E. M. Stein, "Singular integrals and differentiability properties of functions", Princeton Mathematical Series 30, Princeton University Press, Princeton, N.J., 1970.

[10] E. M. Stein, Oscillatory integrals in Fourier analysis, in "Beijing lectures in harmonic analysis" (Beijing, 1984), Ann. of Math. Stud. 112, Princeton Univ. Press, Princeton, N.J., 1986, pp. 307-355. 
[11] E. M. STEIN, "Harmonic analysis: real-variable methods, orthogonality, and oscillatory integrals", Princeton Mathematical Series 43, Monographs in Harmonic Analysis, III, Princeton University Press, Princeton, NJ, 1993.

Leslie C. Cheng:

Department of Mathematics

Bryn Mawr College

Bryn Mawr, PA 19010

U.S.A.

E-mail address: 1cheng@brynmawr.edu

Yibiao Pan:

Department of Mathematics

University of Pittsburgh

Pittsburgh, PA 15260

U.S.A.

E-mail address: yibiao@pop.pitt.edu

Primera versió rebuda el 21 de desembre de 1999, darrera versió rebuda el 31 de gener de 2000. 